\title{
The aging immune system. Abstracts presented at the Masoro-Barshop conference on aging. October 13-15, 2021
}

\author{
Editor: Warren Ladiges
}

Exposure to human alveolar lining fluid drives agedependent transcriptional changes in mycobacterium tuberculosis cell envelope biosynthesis pathways

Anna Allué-Guardia ${ }^{\mathrm{a} *}$, Andreu Garcia-Vilanova ${ }^{\mathrm{a}}$, Angélica M. OlmoFontánez $^{\mathrm{a}, \mathrm{b}}$, Jay Peters ${ }^{\mathrm{c}}$, Diego J. Maselli ${ }^{\mathrm{c}}$, Joanne Turner ${ }^{\mathrm{d}}$, Larry S Schlesinger ${ }^{\mathrm{d}}$, Jordi B. Torrelles ${ }^{\mathrm{a}}$

${ }^{a}$ Population Health Program, Texas Biomedical Research Institute, San Antonio, TX 78227, USA.

${ }^{\mathrm{b}}$ Integrated Biomedical Sciences Program, University of Texas Health Science Center at San Antonio, TX 78229, USA.

${ }^{\circ}$ Host-Pathogen Interactions Program, Texas Biomedical Research Institute, San Antonio, TX 78227, USA.

Mycobacterium tuberculosis (M.tb) is the causative agent of tuberculosis (TB) and one of the top leading causes of mortality worldwide due to a single infectious agent. There were almost 1.4 million attributed TB deaths in 2019 , predicted to increase by $20 \%$ over the next 5 years due to the COVID-19pandemic. After inhalation, M.tb reaches the alveolar space and comes in contact with soluble components of the alveolar lining fluid (ALF) before and after its encounter with host alveolar cells. Our previous work demonstrated that hydrolytic enzymes present in the ALF alter the M.tb cell envelope, driving subsequent $M$.tb-host interactions and infection outcomes in vitro and in vivo. Moreover, the levels and functionality of ALF soluble components are altered in human populations such as the elderly (60+ years old), linked to high local oxidative stress and a proinflammatory environment. In this study, a targeted transcriptional assay demonstrates that exposure to human ALF alters the expression of M.tb cell envelope genes. Further, we show that M.tb exposed to healthy adult ALF (A-ALF) upregulates cell envelope genes associated with lipid, carbohydrate, and amino acid metabolism, as well as other genes related to redox homeostasis and transcriptional regulation, while $M$.tb exposed to dysfunctional ALF from elderly donors (E-ALF) shows a much more limited transcriptional response. Overall, this study indicates that M.tb adapts to the lung alveolar environment upon contact, and that the host ALF status determined by factors such as age may play an important role in determining infection outcome.

Correspondence to: Anna Allué-Guardia, Population Health Program, Texas Biomedical Research Institute, San Antonio, TX 78227, USA.

Email: aallueguardia@txbiomed.org

SToMP-AD: Senolytic therapy to modulate the pro- gression of alzheimer's disease, a vanguard pilot study

Valentina R. Garbarino ${ }^{a}$, Mitzi M. Gonzales ${ }^{\mathrm{b}}$, Eduardo M. Zillib, Ronald C. Petersen ${ }^{\mathrm{c}}$, James L. Kirkland ${ }^{\mathrm{d}}$, Tamara Tchikonia ${ }^{\mathrm{d}}$, Nicolas Musi ${ }^{\mathrm{e}}$, Sudha Seshadri ${ }^{\mathrm{b}, \mathrm{f}}$, Suzanne Craft ${ }^{\mathrm{g}}$, Miranda Orr ${ }^{\mathrm{g}}$

${ }^{a}$ Department of Medicine, Glenn Biggs Institute for Alzheimer's and Neurodegenerative Diseases, UT Health SA, San Antonio, TX 78229, USA .

${ }^{\mathrm{b}}$ Department of Neurology, Glenn Biggs Institute for Alzheimer's and Neurodegenerative Diseases, UT Health SA, San Antonio, TX 78229, USA.

${ }^{\mathrm{C}}$ Department of Neurology, Mayo Clinic, Rochester, MN 55902, USA.

${ }^{\mathrm{d}}$ Robert and Arlene Kogod Center on Aging, Mayo Clinic, Rochester, MN 55905, USA

${ }^{e}$ Department of Medicine, Barshop Institute for Longevity and Aging Studies, San Antonio Geriatric Research Education and Clinical Center (GRECC), UT Health SA, San Antonio, TX 78229, USA.

${ }^{\mathrm{f}}$ Department of Neurology, Boston University School of Medicine, Boston, MA 02118, USA.

${ }^{g}$ Gerontolgy and Geriatric Medicine, Wake Forest School of Medicine, Winston-Salem, NC 27157, USA.

Senescent cells accumulate naturally with age and disease state, and preclinical evidence indicates senescent cell accumulation correlates with pathological tau protein accumulation in Alzheimer's and related tauopathies, indicating tau may be a driver cellular senescence in the brain. Preclinical studies in mouse models of tauopathies demonstrate that a 12-week intermittent treatment with the well-established senolytic therapy combination of dasatinib plus quercetin, reduced tau accumulation, maintained neuronal density, and reduced ventricular enlargement. As a follow-up to these promising preclinical studies, we conducted a small pilot study to establish the safety and examine the efficacy of dasatinib plus quercetin for ameliorating the progression of Alzheimer's disease. Five individuals over 65 with early Alzheimer's disease were enrolled in a 12-week open-label vanguard pilot study. The primary outcomes are to establish the safety profile in this target population through assessment of adverse events, and to confirm study drugs cross the blood-brain-barrier as measured in cerebrospinal fluid. Additional experimental outcomes will be assessed to compare pre- and posttreatment change in cognitive and behavioral outcomes, brain structure (MRI), and markers of pathology in blood and cerebrospinal fluid samples. As an addition to the protocol, we will follow-up with participants three months after study completion to reassess cognitive and blood outcomes to inform longer-term effects before beginning the double-blind placebo-controlled phase II study, 
SToMP-AD, which will follow participant outcomes for 1 year. Gonzales M., et al. Senolytic Therapy to Modulate the Progression of Alzheimer's Disease (SToMP-AD): A Pilot Clinical Trial. 2021. J Prev Alz Dis.

Correspondence to: Mitzi M. Gonzales, Department of Neurology, Glenn Biggs Institute for Alzheimer's and Neurodegenerative Diseases, UT Health SA, 8403 Floyd Curl Drive, MC 8070, San Antonio, TX 78229, USA.

Email: GonzalesM20@uthscsa.edu

Water T-Maze spatial learning test to assess cognitive flexibility in aged mice

Susan Greene ${ }^{a *}$, Gloria Andrea Alcala ${ }^{a}$, Livia Ferriera ${ }^{a}$, Georgianna Gould $^{\mathrm{a}}$

${ }^{a}$ Department of Cellular and Integrative Physiology, Center for Biomedical Neuroscience, University of Texas Health Science Center at San Antonio, San Antonio, TX 78229, USA.

Rodent cognitive flexibility is often assessed through spatial learning in Morris water maze. However, for mice fatigue may introduce a potential confound in these tests, so researchers introduced the water T-maze for mice as a less exhausting alternative spatial learning task (Guariglia SR and Chadman KK. J Neurosci Methods. 2013; 220: 24-9). Fatigue in swim tasks is an important consideration for aged mice, and cognitive flexibility deficits in Morris water maze emerged in aged C57BL6/J mice. We compared performance of 4 each male and female 3,12 and 18 month old mice in water T-maze test, hypothesizing similar deficits to Morris water maze would emerge. For spatial acquisition we filled water T-mazes with 2.5 $\mathrm{L}$ water made opaque by $10 \mathrm{ml}$ white paint. To determine initial preference, each mouse was placed in the maze and the first arm it swam to was noted. A sunken platform was placed in the opposite arm, and the mouse swam in the maze again and time to find the platform and number of incorrect arm entries were noted. This step was repeated for all mice each had 10 rounds of platform acquisition per day for 5 consecutive days. To measure cognitive flexibility, the sunken platform was moved to the opposite from training arm, and the process was repeated. On reversal learning day 1 , the most important day for assessing cognitive flexibility, no differences in performance among ages of mice was observed. This outcome shows water Tmaze is similarly performed by young versus aged mice.

Correspondence to: Susan Greene, Department of Cellular and Integrative Physiology, Center for Biomedical Neuroscience, University of Texas Health Science Center at San Antonio, San Antonio, TX 78229, USA

Email: greenes4@uthscsa.edu

Prepubertal castration reduces the sexually dimorphic elevation of early adulthood mortality in males and improves health parameters in aging genetically heterogeneous mice

Nisi Jiang ${ }^{\mathrm{a}, \mathrm{b}} *$, Catherine J. Cheng ${ }^{\mathrm{a}, \mathrm{b}}$, James F. Nelson ${ }^{\mathrm{a}, \mathrm{b}}$

${ }^{a}$ Department of Cellular and Integrative Physiology, UT Health San Antonio, San Antonio, TX 78229, USA.

Barshop Institute for Longevity and Aging studies, UT Health San Antonio, San Antonio, TX 78229, USA.
Sexual dimorphism in longevity is well recognized in the human population. In the U.S., there is a consistent 5-year difference between males and females in life expectancy. Male mortality rates are markedly higher than those of females, especially in early adulthood. Although driven by the sex chromosomes, the causes of this striking feature are unclear. Genetically heterogeneous (UM-HET3) mice show a similar sexual dimorphism in mortality rates as humans. UM-HET3 mice have higher mortality in males than females, and this elevation, as in humans, is greatest in early adulthood. To investigate the basis for this sex difference, we castrated male UM-HET3 mice before sexual maturation and found that prepubertal castration dramatically reduced the early adult mortality rate of males, although it had no effect on late life mortality. Here we report that prepubertal castration significantly ameliorated the age-related increase in deficit accumulation in older mice, using the Rockwood Frailty Index. Moreover, prepubertal castration delayed the loss of lean mass loss between middle and late ages. These results indicate that some measures of health-span are maintained or improved in prepubertally castrated aging male mice.

Correspondence to: Nisi Jiang, Department of Cellular and Integrative Physiology, UT Health San Antonio, San Antonio, TX 78229, USA.

Email: jiangn@uthscsa.edu

Single-cell transcriptome atlas of the aging mouse pancreatic islet reveals resilience to diet induced obesity

$\mathrm{Jia} \mathrm{Nie}^{\mathrm{a} *}$, Nicolas Musi ${ }^{\mathrm{a}}$

${ }^{a}$ Barshop Institute for Longevity and Aging Studies, University of Texas Health Science Center at San Antonio, San Antonio, TX 78229, USA.

Age is the major contributor to human Type 2 Diabetes (T2D), but the pancreatic islet molecular profile and functional changes during the disease progression are hard to capture in humans. The rodent aging model would mimic the development of T2D in humans. However, the phenotype for the naturally aged rodent is not conclusive, and the association to the onset of T2D in human are not clear. Here, we examine the aging mouse islets by single-cell transcriptomic sampled across young (1 month), middle (13 months), to old (24 months) age groups. The results showed that aging causes a remarkable shift in islet cell composition, diversity, and number, as demonstrated by different markers for each islet cell type. By applying single-cell Western Blot in mouse islets, we further showed that old mice had increased islet beta-cell percentage at both RNA and protein levels, which was accompanied by an increased insulin secretion profile in the islet. Sixmonth high-fat diet feeding was applied to young and old groups to test the resilience to diet-induced obesity in the mice. Surprisingly, the age-associated compensatory hyperinsulinemia status prevented mice from long-term high-fat diet-Induced glucose intolerance. Together, the results from this study fill in the gap between the knowledge of the aging mouse model and the pathogenesis of early-stage human T2D. More importantly, our findings demonstrate that age-dependent hyperinsulinemia protect- 
ed diet-induced obesity by adapting islet compositions.

Correspondence to: Maria Gaczynska, Department of Molecular Medicine, UT Health San Antonio, USA.

Email: gaczynska@uthscsa.edu

Age-associated decrease in thymic B-cell expression of Aire and self-antigen genes impairs T-cell tolerance induction

Meghan A. Rueda ${ }^{\text {** }}$, Sergio Cepeda ${ }^{\mathrm{a}}$, Yangming Xiao ${ }^{\mathrm{a}}$, Manpreet Semwal $^{\mathrm{a}}$, Ellen Kraig ${ }^{\mathrm{a}}$, Ann Griffith

${ }^{a}$ Departments of Biochemistry and Structural Biology, the University of Texas Health Science Center, San Antonio, TX 78229, USA.

Susceptibility to many autoimmune diseases increases with age, however mechanisms linking aging and autoimmunity remain incompletely understood. One hallmark of the aging immune system is atrophy of the thymus, the primary site of $\mathrm{T}$ lymphocyte maturation and selection. Concomitant with atrophy, the expression of tissue restricted self-antigen (TRA) genes in medullary thymic epithelial cells (mTECs) required to tolerize cognate $\mathrm{T}$ cells also declines with age. Recent studies revealed that thymic $\mathrm{B}(\mathrm{tB})$ cells express the transcriptional regulator Autoimmune regulator (Aire), which promotes expression of TRAs, and identified a unique set of TRAs expressed by tBs. We found that expression of Aire and Aire-dependent TRA genes declines with age in murine and human $\mathrm{tB}$ cells, leading to the prediction that $\mathrm{B}$ cell-mediated central T-cell tolerance is impaired in older individuals. To test this, we transferred splenic B cells from young and aged AdBDC transgenic mice (expressing Aire-regulated BDC2.5 antigen) into irradiated hosts reconstituted with BDC2.5 TCR transgenic bone marrow. We find that aging diminishes the capacity of AdBDC B cells to mediate clonal deletion of developing BDC2.5-specific T cells in the thymus. We also used MHCII tetramers and ELISpot to detect $\mathrm{T}$ cells recognizing a TRA expressed in $\mathrm{tB}$ cells, Titin (Ttn), an auto-antigen involved in late-onset myasthenia gravis, in the physiological, polyclonal $\mathrm{T}$ cell population in young and aged mice. Our preliminary results suggest that the reduced capacity of aged tB cells to express Aire also impairs their ability to tolerize selfreactive $\mathrm{T}$ cells in the thymus.

Correspondence to: Meghan A. Rueda, Departments of Biochemistry and Structural Biology, the University of Texas Health Science Center, San Antonio, TX 78229, USA.

Emial: ruedam1@uthscsa.edu

Redox regulation of autophagy in thymic stromal cells and central T-cell tolerance

Semwal MK ${ }^{\mathrm{a}}$, Hester $\mathrm{AK}^{\mathrm{b}}$, Cepeda $\mathrm{S}^{\mathrm{a}}$, Xiao $\mathrm{Y}^{\mathrm{a}}$, Udeaja $\mathrm{C}^{\mathrm{a}}$, Griffith $\mathrm{AV}^{\mathrm{a}}$ ${ }^{a}$ Department of Microbiology and Immunology, School of Medicine, University of Texas Health Science Center at San Antonio, San Antonio, TX 78229, USA.

${ }^{b}$ Howard University College of Medicine, Department of Pharmacology, 520 W Street, NW, Suite 3408, Washington, DC 20059, USA.

Thymic epithelial cells (TECs) are critical regulators of T-cell tolerance, and recent work has revealed that con- stitutive autophagy in TECs is required for self-antigen presentation and negative selection of potentially autoreactive $\mathrm{T}$ cells in the thymus. However, the mechanisms regulating constitutive autophagy in TECs are not well understood. We previously identified conspicuously low levels of the $\mathrm{H}_{2} \mathrm{O}_{2}$ quenching enzyme catalase in thymic stromal cells, and given that $\mathrm{H}_{2} \mathrm{O}_{2}$ promotes autophagy flux, we investigated whether the redox status of these cells established by low catalase expression promotes $\mathrm{T}$ -cell tolerance by enhancing basal autophagy. Transgenic overexpression of catalase diminished autophagy in cTEC and dendritic cells (DC), and decreased clonal deletion among immature $\mathrm{T}$ cells. Catalase overexpression also increased spontaneous lymphocytic infiltrates and increased serum anti-nuclear antigen (ANA) reactivity in 6-8 month old mice. Effects on clonal deletion and autoimmune indicators were generally reversed in catalase transgenic mice when autophagy was rescued by expression of the Becln1F121A/F121A mutant allele. These results suggest a novel metabolic mechanism by which the redox status of thymic stromal cells regulates central T-cell tolerance in the thymus. Supported by NIH grant R01AI121367 and the Max and Minnie Tomerlin Voelcker Young Investigator Award.

Correspondence: Semwal MK, Department of Microbiology and Immunology, School of Medicine, University of Texas Health Science Center at San Antonio, San Antonio, TX 78229, USA.

Email: semwal@uthscsa.edu

Aged mouse model of $\mathrm{C}$. difficile infection: The interplay of aging immunity and microbiome in infection pathogenesis

\section{Jae Hyun Shin ${ }^{a}$, Cirle A. Warren}

${ }^{a}$ Department of Medicine, Division of Infectious Disease and International Health, University of Virginia, Charlottesville, VA 22908, USA.

Clostridioides difficile infection (CDI) poses a serious health issue for the aging population, likely contributing to infectious diarrhea as the only infection-related mortality to increase from 1990 to 2014 in the US. Aged mouse model of CDI has emerged as a valuable tool to evaluate the mechanisms underlying poor outcomes of CDI in the aging host. All published studies utilizing the aged mouse models of CDI uniformly demonstrated more severe disease in aged (around 7 to 28 months old) compared to young ( 2 months old) mice. The most consistent finding demonstrated in every model is a diminished neutrophil recruitment to intestinal tissue in aged mice. Few studies also observed differences in innate (eosinophils, pro-inflammatory cytokines) and humoral (anti-toxin A antibody production) immune responses. Promisingly, the effects of aging on the outcome of infection could be reversed by recombinant IL-22 infusion or fecal microbiota transplant. Fecal microbiota transplantation protects aged mice against deaths. Among the intestinal microbiota, bacteria in the Bacteroidetes phylum appear to play the biggest role in conferring protection, as success of engraftment of the Bacteroidetes phylum seems to correlate closely 
with survival. Components of the host immune response including innate lymphoid cells and IL-22 may correlate with the microbiome changes mediating protection against CDI. Aged mouse model of CDI presents a promising in vivo system to study the effects of aging on immune response to infection and identify potential therapeutics, including microbiota-targeted interventions, specific for the elderly host.

Correspondence to: Jae Hyun Shin, Department of Medicine, Division of Infectious Disease and International Health, University of Virginia, Charlottesville, VA 22908, USA.

Email: jhs9ff@virginia.edu

Impact of asynchronous age-related involution of secondary lymphoid tissues on naive T-cell peripheral maintenance.

Sandip Ashok Sonar ${ }^{a}$, Jennifer Uhrlaub ${ }^{\text {a }}$, Janko Nikolich-Zugich ${ }^{a}$ Department of Immunobiology, University of Arizona College of Medicine, Arizona Center on Aging, Tucson, AZ 85724, USA.

The reduced thymic output and altered peripheral maintenance of TN cells contribute to immune decline in elderly, rendering them more vulnerable to infections, cancer and poor response to vaccination. However, the exact time where these defects occur remains unknown. Using inducible TCR $\delta$ Cre.ER.ZsGreen mouse model, that indelibly marks recent thymic emigrants (RTEs), we longitudinally analyzed the age-related decline of TN cells in SLO during homeostasis. Our data show the early onset (6 months) decline of RTEs in the skin-draining (axillary and inguinal) LNs, while brachial LNs and spleen exhibit delayed (18 months). These defects in the seeding and retention of RTEs are in part due to reduced expression of LN homing chemokine receptor, CCR7 and a transient increase in the surface expression of sphingosine 1-phosphate receptor (S1P1). Interestingly, similar early decline (6 mo) of TN cells observed when we longitudinally monitored genetically-labeled $\mathrm{T}$ cells generated at 2 months of age for lifelong. Interestingly, these timing of RTE maintenance defect correlated well with the LN stromal architecture and organizations. Finally, we demonstrated that the response of RTE to infectious challenge started declining at around 6 mo. Collectively, these results suggest that different SLO undergo age-related changes differently, and warrants mechanistic studies to better understand defects in TN cell peripheral maintenance during aging.

Correspondence to: Sandip Ashok Sonar, Department of Immunobiology, University of Arizona College of Medicine, Arizona Center on Aging, Tucson, AZ 85724, USA.

Email: sandipsonar@e-mail.arizona.edu

An early decline in circulating lymphoid progenitors contributes to the age-associated reduction in ETP numbers

Jayashree Srinivasan $^{\mathrm{a} *}$, Anusha Vasudev ${ }^{\mathrm{b}}$, Hilary J. Selden ${ }^{\mathrm{a}}$, Perez Jr.,Encarnacion ${ }^{\mathrm{b}}$, Bonnie LaFleur ${ }^{\mathrm{c}}$, Shripad Sinari ${ }^{\mathrm{c}}$, Andreas Krueger ${ }^{\mathrm{d}}$, Ellen R. Richie ${ }^{\mathrm{b}}$, Lauren I. Ehrlich ${ }^{\mathrm{a}}$

${ }^{a}$ Department of Molecular Biosciences, Institute of Cellular and Molecular Biology, The University of Texas at Austin, Austin, TX 78712,
USA.

${ }^{\mathrm{b}}$ Department of Microbiology, Immunology \& Molecular Genetics, University of Texas Long School of Medicine, UT Health Science Center, San Antonio, TX 78229, USA.

${ }^{\mathrm{c}}$ Division of Epidemiology and Biostatistics, Mel and Enid Zuckerman College of Public Health, University of Arizona, Tuscon, AZ 85724 5163, USA.

${ }^{d}$ Institute of Molecular Medicine, Goethe University Frankfurt am Main, 60590 Frankfurt am Main, Germany.

Age-associated thymic involution is characterized by decreased generation and export of $\mathrm{T}$ cells, with an associated increase in susceptibility to infections. Elucidating factors responsible for diminished T-cell production during involution will inform strategies to restore T-cell output from the aged thymus. T cells develop from hematopoietic progenitors that migrate from the bone marrow (BM) to the thymus. Early T-cell progenitors (ETPs) require signals from the thymic microenvironment to undergo $\mathrm{T}$ lineage commitment and differentiation. In mice, we find a significant decline in thymocyte cellularity as early as 3 months of age (3MO), which is mirrored by a reduction in ETPs. This early thymic involution could be driven by changes in hematopoietic progenitors and/or thymic stromal cells. Using a multicongenic BM transplantation approach, we determined that the number of ETP-supportive niches does not diminish with age. Notably, the number of lymphoid progenitors in the BM and blood declines dramatically by $3 \mathrm{MO}$, although their cell-intrinsic capacity to seed and differentiate in the thymus is unimpaired, suggesting that pre-thymic hematopoietic changes contribute to the early decline in ETPs. However, modulating the thymic stromal compartment sustains ETP cellularity, supporting the idea that age-associated defects in ETP niche quality also contribute to the reduction in ETPs. Consistent with this possibility, Notch signaling, which is essential for T-lineage differentiation, diminishes significantly in $3 \mathrm{MO} \mathrm{BM}$ progenitors and thymic ETPs. Together, our findings suggest that the early age-associated decline in ETPs is due to both hematopoietic and stromal changes. Supported by P01 AG052359 from the NIH-National Institute on Aging.

Correspondence to: Jayashree Srinivasan, Department of Molecular Biosciences, Institute of Cellular and Molecular Biology, The University of Texas at Austin, Austin, TX 78229, USA.

Email: jayashree@utexas.edu

Age-dependent changes in the regulatory program of CD8 regulatory $\mathrm{T}$ cells (CD8 Treg)

Saranya Srinivasan ${ }^{\text {a* }}$, Nu Zhang

${ }^{a}$ Department of Microbiology, Immunology and Molecular Genetics, The Joe R. and Teresa Lozano Long School of Medicine, University of Texas Health Science Center at San Antonio, San Antonio, TX 78229, USA.

Regulatory T cells help maintain the immune homeostasis by tolerance to self-antigens. Any defect in the regulatory $\mathrm{T}$ cells causes dysregulated immune responses that leads to risk of infection, autoimmunity and cancer. And the risk increases with age significantly since elders are severely affected during infections due to ineffective 
immune response also making vaccines less effective. Increased morbidity in the elderly patients is due to agerelated changes in the immune system called immune aging. Understanding the immune-aging is critical to intervene in age-associated immune diseases. Despite several age-related studies in immune cells, immune aging in CD8 Tregs are poorly characterized. The primary focus of this work is to study the age-associated changes in the CD8 Tregs. In this study, we did a homeostatic quantitative and qualitative analysis of CD8 Treg in young and older mice. We used acute infection model to test the agerelated effect of infection/inflammation in the CD8 Treg. Our results demonstrate that at homeostasis frequency of CD8+ Tregs in total lymphocytes is higher in older mice and further expanded during acute infection. Interestingly, CD8 Treg in aged mice is functionally superior to young mice and exhibit dramatically altered metabolic profiles. Our findings support that CD8 Treg may contribute to the weakened immune response in the elders.

Correspondence: Saranya Srinivasan, Department of Microbiology, Immunology and Molecular Genetics, The Joe R. and Teresa Lozano Long School of Medicine, University of Texas Health Science Center at San Antonio, San Antonio, TX 78229, USA.

Email: srinivasons@livemail.uthscsa.edu

T-bet $+B$ cells promote adipose tissue inflammation and metabolic disorder during obesity

Carlo Vanz ${ }^{\mathrm{a} *}$, Thomas Hägglöf $f^{\mathrm{a}}$, Elizabeth Dudley ${ }^{\mathrm{a}}$, Elizabeth A ${ }^{\mathrm{a}}$, Leadbetter $^{\mathrm{a}}$

${ }^{a}$ Department of Microbiology, Immunology \& Molecular Genetics, University of Texas Health Science Center at San Antonio, San Antonio, TX 78229 , USA.

Obesity is a major health crisis affecting a large and evergrowing portion of the global population. Being obese is associated with increased mortality and a lower quality of life, and it also puts an increased economic burden on healthcare systems. Obesity is linked with a low-grade and chronic inflammation that originates in the adipose tissue and further exacerbates systemic metabolic dysfunctions. Understanding the mechanisms and immune cell populations promoting this inflammation would allow for the development of new therapies to restore immune regulation in obese patients. Our lab has identified a subset of B lymphocytes which is expanded in the adipose tissue of humans and mice during obesity. These B cells co-express the transcription factor T-bet and the integrin CD11c, they produce IgG2c antibodies, and their expansion is dependent on the presence of iNKT cells. In vivo studies with mice fed a high fat diet reveal that these Tbet + B cells promote inflammation. Serum transfer experiments and flow cytometry analysis revealed that this effect is mediated through the production of CXCL10 and antibodies. The genetic ablation of T-bet $+\mathrm{B}$ cells does not appear to affect adipose CD4+ T cells or NK cells, but it reduces the proinflammatory polarization of adipose tissue macrophages. Transgenic mice engineered to lack T-bet + $B$ cells not only have reduced adipose tissue inflammation, but present improved metabolic function. Based on these results, T-bet $+\mathrm{B}$ cells represent an interesting potential target to reduce inflammation in obese patients and to improve their metabolic functions.

Correspondence to: Carlo Vanz, Department of Microbiology, Immunology \& Molecular Genetics, University of Texas Health Science Center at San Antonio, San Antonio, TX78229, USA.

Email: vanz@livemail.uthscsa.edu

The aging immune system drives senescence and dysfunction of peripheral tissues

Matthew J. Yousefzadeh ${ }^{\mathrm{a}, \mathrm{b} *}$, Rafael Flores ${ }^{\mathrm{a}, \mathrm{b}}$, Paul D. Robbins ${ }^{\mathrm{a}, \mathrm{b}}$, Laura J. Niedernhofer ${ }^{\mathrm{a}, \mathrm{b}}$

${ }^{a}$ Institute on the Biology of Aging and Metabolism, University of Minnesota, Minneapolis, MN 55455, USA.

${ }^{\mathrm{b}}$ Department of Biochemistry, Molecular Biology \& Biophysics, University of Minnesota, Minneapolis, MN 55455, USA.

The progressive and age-related decline in immune function known as immunosenescence ameliorates the immune response to pathogens or vaccinations in elderly individuals, leaving them susceptible to infections. To study the aged immune system and its effects on the rest of the organism, we prematurely aged the immune compartment by deleting a critical DNA repair gene. Using the codon-improved cre recombinase under control of the Vav1 promoter, Ercc1 was specifically deleted in hematopoietic cells of the mouse (Vav-iCre+/-; Ercc1-/ fl) to increase spontaneously occurring endogenous DNA damage and oxidative stress. Vav-iCre+/-; Ercc1-/fl mice were healthy into adulthood but were observed to have attrition and senescence of immune cell populations as they age. The mice had accelerated and progressive onset of immunosenescence with deficits in B, T, and NK cell function, analogous to what is observed in naturally aged mice. Surprisingly, evidence of increased senescence and oxidative stress in non-lymphoid organs was present, resulting in premature aging of liver, kidney and intervertebral disc and reduced repair capacity of injured muscle. Vav-iCre+/-; Ercc1-/fl mice also had a shortened lifespan, indicating that senescent, aged immune cells potent contributors to systemic aging. Furthermore, transplantation of splenocytes from Vav-iCre+/-; Ercc1-/fl or aged wildtype mice induced senescence in multiple tissues in trans. Meanwhile, transplantation of young splenocytes into Ercc1-/ $\Delta$ progeroid mice reduced senescent cell burden. This work shows that systemic aging can be enhanced by an aged, senescent immune system and highlights the importance of a robust immune system to extend healthy aging.

Correspondence to: Matthew J. Yousefzadeh, University of Minnesota, 321 Church St. SE, JACH 6-155, Minneapolis, MN 55455, USA. Email:myousefz@umn.edu

Cite this article as: Ladiges W, editor. The aging immune system. Abstracts presented at the Masoro-Barshop conference on aging; 2021 Oct 13-15; Virtual, Zoom Webinar, USA. Ant publishing; 2021, 3(4): 79-83. 